\title{
Do Fluency-Induced Pupillary Responses Reflect Aesthetic Affect?
}

\author{
Sophie G. Elschner, Ronald Hübner, and Michael Dambacher \\ Universität Konstanz
}

\begin{abstract}
Recently, Kuchinke, Trapp, Jacobs, and Leder (2009) used pupillary peak dilations (PDs) to test the hypothesis that fluent picture processing elicits aesthetic affects. They used reproductions of cubist pictures of different abstractness as stimuli, which was assumed to modulate processing fluency. As a result, less abstract pictures were not only processed more fluently and preferred, they also produced larger PDs than more abstract ones. This was interpreted as support of their hypothesis. The aim of the present study was to replicate and generalize these results with an improved method and by adding expressionist pictures, which covered a relatively large range of abstractness. In the first experiment, where art style was blocked, there were no clear results. Therefore, the authors randomized art style in the second experiment. This time PDs increased with decreasing abstractness, even though significantly only for the expressionist pictures. However, there was no relation between preference and PDs. Thus, although they also observed a covariation between abstractness and pupil size, the data do not support the idea that PDs reflect fluency-induced aesthetic affect.
\end{abstract}

Keywords: aesthetic preference, emotion, pupil size, processing fluency

The question of why we like some pictures or objects more than others has been debated in philosophy for hundreds of years and was one of the first investigated in experimental psychology (Fechner, 1876). Whereas some theorists assumed that aesthetic preference is determined by object properties such as symmetry, balance, proportion, and prototypicality (cf. Palmer, Schloss, \& Sammartino, 2013), others proposed a subjectivist view, that is, that aesthetic experience depends on the qualities of the perceiver (Reber, Schwarz, \& Winkielman, 2004). Obviously, these two accounts do not exclude each other. Accordingly, recent models have integrated both views into a single model (e.g., Leder, Belke, Oeberst, \& Augustin, 2004).

A prominent mechanism demonstrating the dependency of aesthetic preference on the quality and state of the perceiver is processing fluency (Reber et al., 2004; Winkielman, Schwarz, Fazendeiro, \& Reber, 2003). It is assumed that a stimulus that can be processed fluently by an observer produces a positive affect because it signals successful recognition and error-free processing. Most interestingly, high fluency usually also produces positive evaluations of the processed object.

An important question in this respect is whether affective responses are indeed elicited by fluent processing and, if so, to what extent they are involved in aesthetic judgments, especially for artworks. Recently Kuchinke et al. (2009) tried to answer this question using pupillary responses. Research shows that pupillometry can well be used to examine emotional responses, because pupils dilate if people are excited (Bradley, Miccoli, Escrig, \& Lang, 2008; Henderson, Bradley, \& Lang, 2014; Partala \& Surakka, 2003; Siegle, Ichikawa, \& Steinhauer, 2008; Võ et al., 2008). Indeed, in several studies it was demonstrated that there is a close relation between aesthetic preference and pupil size. Johnson, Muday, and Schirillo (2010), for instance, found that pupil size increased with the pleasantness of paintings. Other researchers found similar results (Blackburn \& Schirillo, 2012), or, if unpleasant pictures were also included in the stimulus set, observed a U-shaped relationship (Bradley et al., 2008; Hayes, Muday, \& Schirillo, 2013; Powell \& Schirillo, 2011).

Kuchinke et al.'s (2009) study is pioneering, because, to our knowledge, it is the first that combined research on processing fluency with pupillometry in the field of experimental aesthetics. They used reproductions of cubist paintings as stimuli and asked their participants to classify the depicted objects. To modulate processing fluency, they presented pictures of different abstractness (for examples see Figure 1). As expected, objects in less abstract pictures were recognized faster, which confirmed that these stimuli were processed more fluently. Furthermore, less abstract pictures were preferred to abstract ones. Most importantly, though, the observed pupil dilations were also larger for less abstract pictures, which suggests that pupil size reflects differences in fluency. Further, a significant item-based correlation between pupil size and preference $(r=.333)$ also implies a relationship between aesthetic affect and pupil response.

Because Kuchinke et al.'s (2009) result is promising, it is important to know whether it is replicable and can be generalized to pictures of other art styles. The present study addressed this issue by conducting a similar experiment, where, in addition to the 


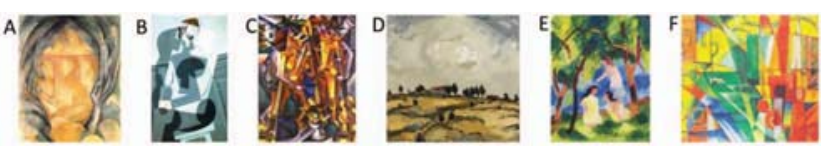

Figure 1. Example stimuli of low (A; Viaduct at l'Estaque, by G. Braque, 1908). Adapted from "Pupillary Responses in Art Appreciation: Effects of Aesthetic Emotions", by Kuchinke et al., 2009, Psychology of Aesthetics, Creativity, and the Arts, 3, 156-163. Copyright 2017 by VG Bild-Kunst, medium (B; Portrait of Josette Gris, by J. Gris, 1916), and high (C; Composition with Figures, L. Popova, 1913) abstract cubist pictures, and of low (D; Autumn Landscape with Clouds, I. Aalto, 1917), medium (E; Girls Bathing, A. Macke, 1913) and high (F; Landscape with House, Dog and Cattle, F. Marc, 1914) abstract expressionist paintings. See the online article for the color version of this figure.

original selection of cubist pictures, reproductions of expressionist paintings were also presented. Cubist painters divide picture objects into basic geometrical forms and show the same object from different viewpoints at the same time. As consequence, cubist pictures are usually rather abstract. Indeed, the mean abstractness ratings for the pictures used by Kuchinke et al. (2009) varied only over a small range. In contrast, expressionist painters express their emotional responses to the depicted objects (Thomas, 2000), which also allows them to depict objects less abstractly. Accordingly, these pictures can vary from very figural illustrations to highly abstract forms, therefore covering a larger range of abstractness and aesthetic preference. If, as assumed by Kuchinke et al. (2009), the abstractness of a picture determines how fluently it can be processed, and if fluency elicits aesthetic emotions, then pupil size should also vary over a larger range, compared to the cubist art style. Because of this property, we also included expressionist pictures as stimuli.

As another critical step, we improved the procedure to eliminate potential confounds in the pupillometric results. In particular, we identified three methodological shortcomings in Kuchinke et al.'s (2009) study. First, there was no control for the initial light reflex of the pupil, which takes place within the first $2 \mathrm{~s}$ after monitor luminance changes from a uniform background to the stimulus picture. Therefore, it is possible that the pupil response to stimulus onset overlapped with the emotional pupil response to the explicit classification task (Beatty \& Lucero-Wagoner, 2000; Henderson et al., 2014). This is especially critical in Kuchinke et al.'s (2009) study, because they assessed pupil dilations relative to the participants' manual responses signaling object recognition (i.e., response-locked), which were considerably faster for pictures of low compared to high abstractness. Consequently, the distortion caused by the initial pupil reflex, were larger for less abstract paintings. In the present experiments, we minimized this problem by adjusting display luminance to that of the critical stimulus before stimulus onset.

Second, we were concerned with the control of luminance across abstractness conditions. Kuchinke et al. (2009) used the toolbox for MATLAB (Malo \& Luque, 2002) to match luminance for picture categories. However, this toolbox does not take the apparent luminance of the stimuli into account (Loewenfeld \& Loewenstein, 1993). Pictures with the same "luminance" values can therefore yield different pupil responses due to differences in the color spectrum and different distributions of light and dark areas (Bradley et al., 2008; Woodmansee, 1966). In fact, Powell and Schirillo (2011) even argued that it is impossible to standardize pictures' luminance. In the present study, this problem was addressed by presenting a scrambled version of the stimulus before the actual stimulus: The pupil adaptation to the critical luminance prior to the onset of a stimulus grants a neutral baseline across stimulus categories, independent from the absolute luminance of each item. Furthermore, we measured the luminance of each stimulus with a photometer to check that stimuli did not differ substantially between the abstractness and art conditions.

A third concern was that Kuchinke et al. (2009) collected preference ratings after their participants had gone through the pupillometric test phase. A pilot study in our lab with the same stimulus material revealed, however, that prior exposure to the stimuli strongly affected subsequent ratings. Specifically, we found no significant effect of abstractness (fluency) on preference $(p=.536)$. This null effect presumably resulted from the rather small variation of preference, which could have been due to a mere exposure effect. Mere exposure facilitates the processing of stimuli with higher abstractness (Reber et al., 2004; Winkielman \& Cacioppo, 2001; Winkielman et al., 2003), increases liking, and decreases disliking regardless of valence (Bornstein, 1989; Reber, Winkielman, \& Schwarz, 1998; Zajonc, Markus, \& Wilson, 1974). Thus, mere exposure could have reduced the differences in liking between pictures of different abstractness. A possible solution to this problem is to obtain preference ratings directly after the explicit classification task for each stimulus. This, however, would have produced task-switching effects (Kiesel et al., 2010) with unpredictable consequences on both the recognition task and the preference rating. Therefore, we decided to collect pupillometric data and preference ratings from separate participants.

\section{Experiment 1}

The aim of this experiment was to replicate and extend the results of Kuchinke et al. (2009). Therefore, we used a similar method including some methodical improvements and used expressionist pictures in addition to cubist pictures as stimuli.

\section{Method}

Before we describe the method of Experiment 1, we report methods and results of preliminary studies in our lab that served to measure stimulus attributes and to select an appropriate set of expressionist pictures.

Stimuli. The cubist stimuli used in the present experiment were the same 39 pictures (for examples see Figure 1) as used in Kuchinke et al. (2009), which are categorized into three levels of abstractness (low, medium, or high). To obtain corresponding expressionist stimuli we collected, in a first step, 60 pictures and asked 63 participants (mean age 24 years, 15 male) to rate them on 5 -point scales with respect to complexity, ranging from 1 (low) to 5 (high); familiarity, ranging from 1 (not familiar) to 5 (highly familiar); and abstractness, ranging from 1 (representational) to 5 (abstract'). The abstractness ratings were then used to select 13 expressionist pictures (for examples, see Figure 1) for each of the categories of low, medium, and high abstractness (see Table 1). As expected, the range of abstractness across the categories was considerably larger for the expressionist pictures than for the cubist ones (see Table 1) reported by Kuchinke et al. (2009). 
Table 1

Mean Stimulus Features for Each Condition of Abstractness and Art Style

\begin{tabular}{lccccc}
\hline $\begin{array}{c}\text { Art style and } \\
\text { abstractness } \\
\text { category }\end{array}$ & Preference & Familiarity & Abstractness & Complexity & $\begin{array}{c}\text { Luminance } \\
\left(\mathrm{cd}^{2} \mathrm{~m}^{2}\right)\end{array}$ \\
\hline $\begin{array}{l}\text { Expressionist } \\
\text { Low }\end{array}$ & 3.00 & 1.49 & & & \\
Medium & 2.76 & 1.43 & 2.61 & 2.29 & 52.3 \\
High & 2.52 & 1.50 & 4.33 & 2.43 & 57.3 \\
Cubist & & & & 3.85 & 54.8 \\
Low & $2.38(3.14)$ & 2.71 & $3.46(3.00)$ & 3.02 & 52.4 \\
Medium & $2.43(2.87)$ & 2.71 & $3.87(3.25)$ & 3.84 & 64.4 \\
High & $2.27(2.80)$ & 2.57 & $4.41(3.69)$ & 4.30 & 61.5 \\
\hline
\end{tabular}

Note. Values for abstractness are from our re-evaluation study. For the cubist stimuli, the values from Kuchinke et al. (2009) are shown in parentheses. Values for familiarity and complexity for the cubist pictures were taken from Kuchinke et al. (2009). Luminance was measured with a photometer.

To rule out that the different ranges of abstractness between the art styles were due to methodological differences between the two studies rather than to the stimuli, we conducted an online study, in which 55 persons rated the abstractness of the mixed pictures from both art styles. Five participants were excluded from data analysis, because their ratings were incomplete. The result of the remaining 50 participants (mean age $=23.5$ years, 6 male) confirmed our previous results. Although the range of abstractness for the cubist pictures was somewhat larger than that reported in Kuchinke et al. (2009), it was still considerably smaller than that for the expressionist pictures (see Table 1, and Figure 2). Analyses of variance (ANOVAs) revealed a significant difference in abstractness between the art styles, $F(2,98)=592, p<.001$.

The COLORLAB toolbox for MATLAB (Malo \& Luque, 2002) was used to standardize the luminance between the abstractness conditions for the expressionist pictures. Subsequent measurements with a photometer (Tektronix, Lumacolor II) revealed that the luminance did not differ significantly between the art styles, $F(1,72)=0.990, p=.323 ; \eta^{2}=.013$, and between the abstract-

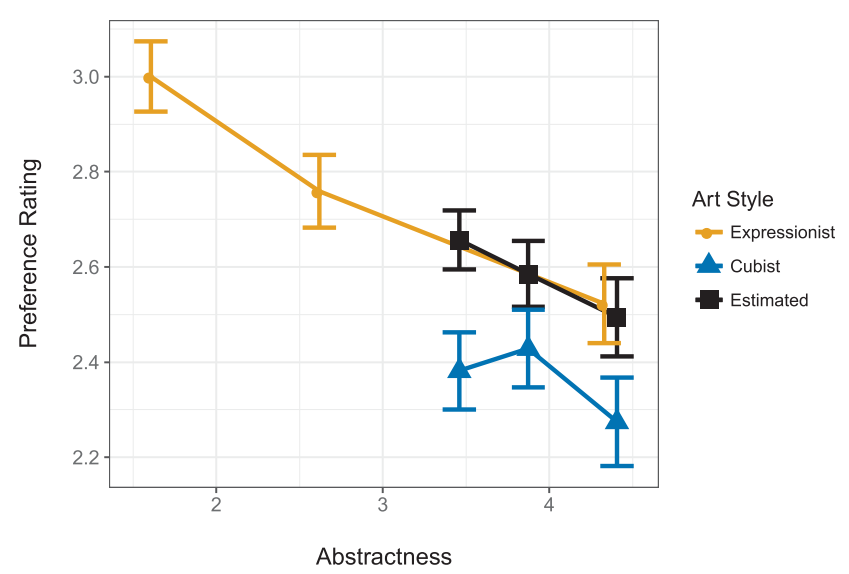

Figure 2. Results of the preference ratings for the two art styles. The black squares represent estimated values (by linear regression) of expressionist stimuli that correspond to the range of abstractness values of the cubist stimuli. Error bars represent standard errors. See the online article for the color version of this figure. ness levels $F(2,72)=1.15, p=.323 ; \eta^{2}=.030$. The different ratings and measurements are shown in Table 1.

In a further step, we asked 56 persons in an online survey to rate the 39 cubist and 39 expressionist pictures on a 5-point scale with respect to preference, ranging from 1 (not at all) to 5 (very much). As incentive, participants could take part in a tombola with the prospect to win €20. Data from three participants were excluded, because they were art experts. ${ }^{1}$ The remaining 53 participants were aged 17 to 34 years $(M=22.42, S D=2.72 ; 16$ male).

In Figure 2 (see also Table 1), it can be seen that preference varied linearly with abstractness for the expressionist stimuli. For the cubist stimuli, the relation was nonlinear, which differs from result in Kuchinke et al. (2009). To test whether the relations were significant, we computed a one-way ANOVA for each art style, with the within-participant factor abstractness (low, medium, or high). For the expressionist stimuli, it revealed a significant effect, $F(2,104)=14.3, p<.001 ; \eta^{2}=.108$. Bonferroni corrected $t$-tests showed that the high and low abstractness levels differed significantly $(p<.05)$. For the cubist stimuli there was also a significant effect of abstractness, $F(2,104)=4.47, p<.05 ; \eta^{2}=$ .011 , which was due to a significant difference between the medium and high level of abstractness.

Correlational analyses. An item-based correlational analysis across the pictures of both art styles confirmed the significant relation between abstractness and preference $(r=-.484 ; p<$ $.001)$. With respect to the individual art styles, it was significant for the expressionist pictures $(r=-.394 ; p<.05)$, and marginally significant for the cubist ones $(r=-.263 ; p=.058)$.

To test whether the difference in preference between the art styles was significant, it would have been inappropriate to include the obtained data directly into a common ANOVA, because of the range difference in abstractness between the art styles. Therefore, we applied linear regression to the expressionist data to estimate the preference ratings that correspond to the abstractness values of the respective cubist categories for each participant. The mean estimated values are represented by the black squares in Figure 2. The data for the cubist pictures and the corresponding estimated

\footnotetext{
${ }^{1}$ Art expertise was assessed via self-report on the participant's field of study. Data from participants who studied in the university's literature-artmedia program were excluded from analyses.
} 
data for the expressionist pictures were then entered into a repeated-measures one-way ANOVA with art style as factor. It revealed a significant effect, $F(1,52)=11.24, p=.002 ; \eta^{2}=$ .035. Thus, even if we restrict the comparison to their common range of abstractness, our data reveal a trend toward greater preference for expressionist rather than for cubist pictures, although the effect was small.

To control for possible confounds between pupil size and picture abstractness, caused by the initial pupil reflex, scrambled versions of the pictures were created in MATLAB by shuffling the pixels of each picture. In the experiment, the pictures were presented on a computer screen with a gray background (RGB: 200, $200,200)$. The visual angle of the pictures ranged from $8.97^{\circ}$ to $15.22^{\circ}$ vertically and from $7.20^{\circ}$ to $14.74^{\circ}$ horizontally.

For the required experimental task, the pictures of both art styles contained either human figures or a landscape. Five additional pictures with other content (still lifes) were chosen for each style to ensure that participants could not anticipate the picture's content.

Participants. Forty-seven volunteers at the Universität Konstanz were recruited via ORSEE (Greiner, 2015) and participated in the pupillometric experiment. None of them had participated in the rating studies. Data of 10 participants were excluded from analysis, because they were substantially older than the average of the sample, because of technical problems during recording, or because more than $50 \%$ of their pupillary data were missing after artifact rejection. The remaining 37 participants (14 male) were art novices, aged between 19 and 32 years $(M=23.78$; $S D=3.44)$. They had normal or corrected-to-normal vision, with the exception of one participant who had -1 diopter in the left eye and -0.5 diopter in the right eye by self-report, which, however, did not affect data quality.

The experiment was performed in accordance with the ethical standards laid down in the 1964 Declaration of Helsinki and its later amendments. In agreement with the ethics and safety guidelines at the Universität Konstanz, we obtained a verbal informed consent statement from all individuals prior to their participation in the study. Potential participants were informed of their right to abstain from participation in the study or to withdraw consent to participate at any time without reprisal. The participants received either $4 €$ or course credit for participation in the 30-min session.

Procedure. The participants were seated in front of a computer screen with their head positioned on a chin rest, such that the distance between monitor and eyes was $60 \mathrm{~cm}$. Pupil diameters were recorded with a video-based infrared eye tracker (SensoMotoric Instruments, SMI, RED500; Berlin, Germany) at a sampling rate of $500 \mathrm{~Hz}$. The eye tracker was calibrated for both eyes on a five-point grid at the beginning of an experimental session. The experiment was controlled via Presentation 16.4 (Neurobehavioral Systems Inc., Berkeley,CA), and pupil recording and trial presentation were synchronized with the iView $\times 2.8$ interface. The experiment started with a written instruction followed by three practice trials with impressionistic pictures. Participants were instructed to press the left button on a USB mouse as quickly as possible when they had identified an object in the picture. Each trial started with a fixation cross at the center of the screen that lasted for 1,000 ms as a standard. A fixation check was included that initiated a trial only if the eye tracker had detected a valid fixation on the fixation cross within $5,000 \mathrm{~ms}$; fixation check failure entailed a new calibration. After a scrambled version of the picture, which was shown for $2.5 \mathrm{~s}$ to elicit the initial light reflex (Henderson et al., 2014), the stimulus picture was presented and remained on the screen until $1.0 \mathrm{~s}$ after the response, or for a maximum of $10 \mathrm{~s}$, if the participant failed to identify an object. Each trial ended with the presentation of a smiley for $3 \mathrm{~s}$. During this time interval, the participants were allowed to blink (see Figure 3). A control question (i.e., "In one word: Please describe what you saw on the picture") was presented after a random selection of trials to make sure that participants followed the task instructions.

Cubist and expressionist pictures were presented in two separate blocks with a short break in between. Twenty participants worked through the cubist block first, and then through the expressionist block, whereas the order was reversed for the other 17 participants. In each block, the pictures were presented in random order.

Data processing. Programs written in MATLAB (R2014b) and $\mathrm{R}$ ( $\mathrm{R}$ Core Team, 2015) were used to process the pupillometric data from the left eye (Loewenfeld \& Loewenstein, 1993; Powell $\&$ Schirillo, 2011). Continuous raw data were down sampled to $100 \mathrm{~Hz}$, and epochs were defined between $1 \mathrm{~s}$ before and $10 \mathrm{~s}$ after stimulus onset. A 7-point weighted average filter was employed to smooth the data. Practice trials, filler trials, epochs with major blinks, or trials without response were discarded. Likewise, trials with responses faster than $100 \mathrm{~ms}$ were excluded. Finally, data were visually checked for artifacts. In total, $26 \%$ of all trials (ranging from $8 \%$ to $47 \%$ across participants) were discarded. For each epoch, response-locked pupil data were adjusted relative to a $200 \mathrm{~ms}$ preresponse baseline. The maximum pupil diameter in the interval between the participant's response (i.e., button press) and $1 \mathrm{~s}$ thereafter was then taken as peak dilation (PDs).

Different from the pupil data, trials with blinks were included in the response time (RT) analyses. Only trials with responses faster than $100 \mathrm{~ms}$ or without response were discarded (8\%). All further analyses were done with R 3.2.0 (R Core Team, 2015), and the results were visualized with ggplot2 (Wickham, 2009).

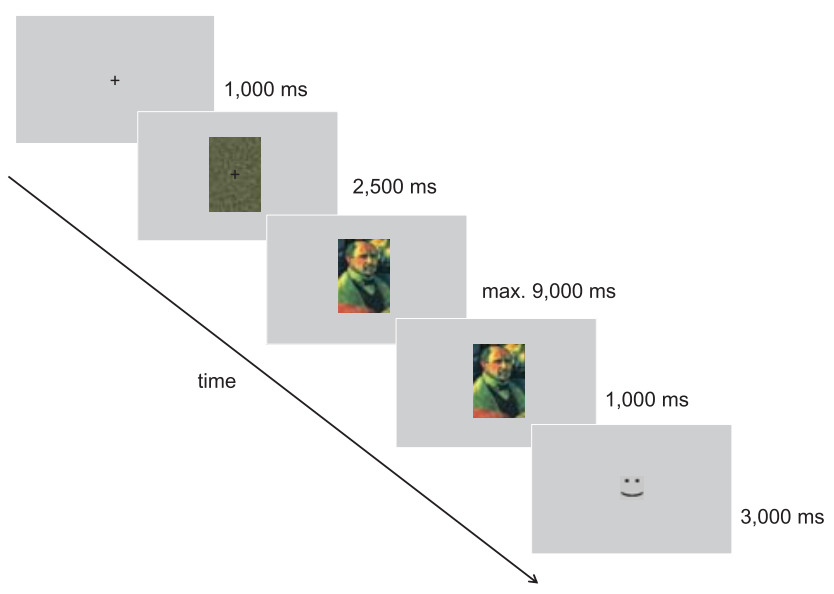

Figure 3. Standard trial procedure. Time specifications show the duration of each slide within one trial. The painting is Self-Portrait, by A. v. Jawlensky, 1912. Vienna, Austria: Österreichische Galerie Belvedere. See the online article for the color version of this figure. 


\section{Results and Discussion}

Response times. Because the range of abstractness largely differed between the art styles (see Table 1), entering the mean RTs for both art styles into a common ANOVA would have been inappropriate. Therefore, we analyzed the data separately for each art style by a one-way ANOVA with abstractness (low, medium, or high) as within-participant factor. Abstractness had a significant effect for the expressionist pictures, $F(2,72)=59.7, p<.001$; $\eta^{2}=.252$, as well as for the cubist ones, $F(2,72)=61.7, p<$ $.001 ; \eta^{2}=.195$. As can be seen in Figure 4 (see also Table 1), for both art styles RTs increased with abstractness. Subsequent Bonferroni-corrected $t$-tests revealed that for both art styles RTs differed significantly (all $p<.05$ ) between all abstractness categories, except between expressionist pictures of low and medium abstractness.

Correlational analyses. An item-based correlational analysis produced analogous results. Across pictures and art styles it revealed a significant relation between abstractness and RT ( $r=$ .794; $p<.001)$. The correlations were also significant for the subsets of expressionist pictures $(r=.849 ; p<.001)$ and cubist pictures $(r=.857 ; p<.001)$.

Our RT data show that the time needed for recognizing an object increased with the abstractness of the stimulus. As Kuchinke et al. (2009), we interpret this result in the sense that the abstractness of our pictures determined the fluency of processing. Although RTs might not generally reflect fluency (Oppenheimer, 2008), given the participants' task, it seems reasonable to assume that it did in this experiment.

Pupillary dilations. Figure 5 shows the response-locked time course of the pupil size for the different experimental conditions. As can be seen, pictures of low abstractness led to a substantial increase in pupil size, compared to the other abstractness levels. For pictures of high and medium abstractness, the difference was less pronounced. Notably, the order did not correspond to our expectation. For each art style, they were highest for the lowest abstractness category, but lowest for the medium category.

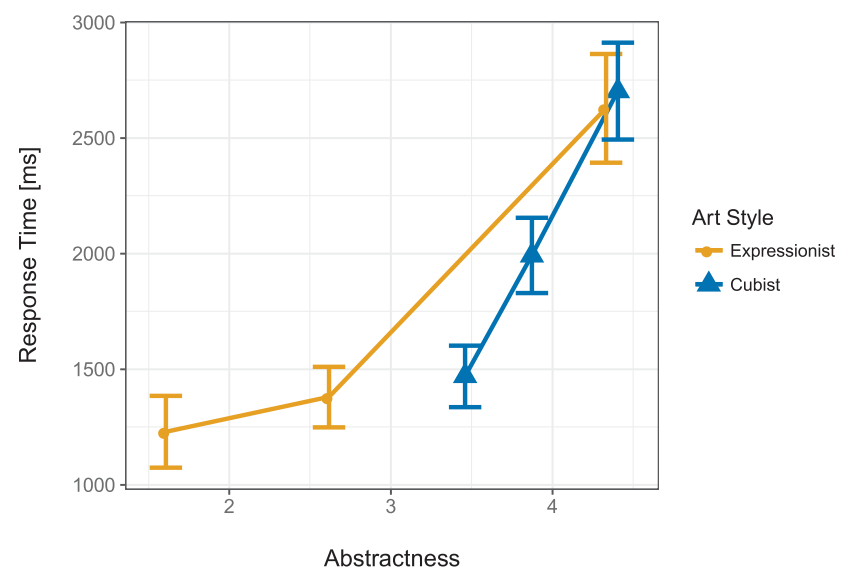

Figure 4. Response times for recognizing an object in cubist and expressionist pictures as a function of abstractness in Experiment 1. Error bars represent standard errors. See the online article for the color version of this figure.
To test whether the effects of abstractness were significant within the art styles, PDs for each art style were subjected to one-way ANOVAs with abstractness (low, medium, or high) as within-participant factor. The effect of abstractness was significant for the expressionist pictures, $F(2,72)=5.43, p<.01$; $\eta^{2}=.023$, as well was for the cubist ones, $F(2,72)=4.83, p<$ $.05 ; \eta^{2}=.023$. As can be seen in Figure 6 (see also Table 2), low abstractness produced higher PDs than the other two levels, which did not differ significantly. Notably, against the predicted relation between abstractness and pupillary responses, PDs were generally numerically larger for cubist than for expressionist paintings.

Correlational analyses. An item-based correlational analysis across pictures and art styles revealed that there was no significant overall relation between abstractness and $\mathrm{PD}(r=-.100 ; p=$ $.382)$. For the individual styles, the correlation was not significant for the expressionist pictures, $(r=-.180 ; p=.272)$, and marginally significant for the cubist ones, $(r=-.306 ; p=.058)$. Because RT can be considered as further index of fluency, we also computed the correlation between RT and PD. Overall, it was not significant, $(r=-.057 ; p=.622)$. This also held for cubist, $(r=-.085 ; p=.605)$ and for expressionist, $(r=-.100 ; p=$ .547) pictures alone. In contrast, there was a significant overall correlation between preference and PD, $(r=-.271 ; p<.05)$. However, its direction was opposite to the expectations: greater preferences came with smaller pupil size. For the individual styles the correlation was in the same opposite direction, but not significant (cubist: $[r=-.259 ; p=.111]$; expressionist: $[r=-.226$; $p=.167])$.

Thus, given the strong relation between abstractness and preference, as found in our picture ratings, and the corresponding relation between abstractness and RTs in the task of the present experiment, the observed PDs do not support the idea that fluency-induced affective aesthetic responses are reflected by pupil size. As the ANOVAs revealed, within each art style, there was an increase in PDs only between the highest and the two lower abstractness categories. Between the lower categories, there was no substantial difference. The trend was even opposite to the expected direction. The correlational analyses also revealed no significant relation between PD and abstractness or RT. The only significant correlation occurred between PD and preference, which, however, indicated that pictures of higher preference produced smaller pupil size. This is opposite to what we expected.

Finally, cubist paintings, which were liked less than expressionist pictures, produced numerically higher PDs. This is also incompatible with the idea that preference is positively related to pupil size.

Sequential block effects. When we looked for possible reasons why cubist pictures produced a larger pupil size than expressionist stimuli, we also considered the data for the two art-style sequences separately. As shown in Figure 7, PDs for cubist pictures were larger for participants who processed these stimuli first. When cubist pictures were processed after expressionist ones, the PDs were smaller. Figure 7 also suggests that starting with cubist pictures had a greater damping effect on the subsequent PDs for expressionist pictures than vice versa. Apparently, effects such as practice, fatigue or motivation through the course of the experiment critically distort pupillary responses and have to be taken into 


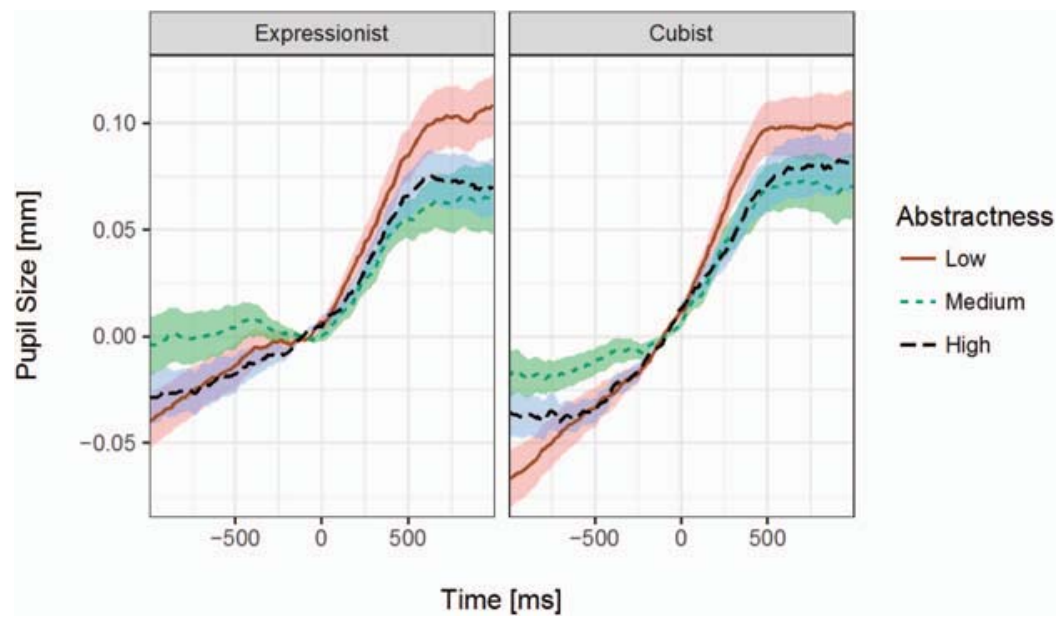

Figure 5. Response-locked time course of the pupil size (in $\mathrm{mm}$ ) for the art styles and abstractness conditions in Experiment 1. Ribbons around the graphs represent standard errors. See the online article for the color version of this figure.

account as confound. Thus, to avoid such sequential effects, we conducted a second experiment, where the art styles were randomly mixed.

\section{Experiment 2}

This experiment was identical to the first one, except that art styles were mixed rather than sequentially blocked. We expected that, due to the style mixing, our participants would similarly process both art styles and thus apply the same response criterion. The hypotheses were adopted from Experiment 1.

\section{Method}

Participants. Twenty-five students from the Universität Konstanz were recruited. The data of five participants were excluded from analyses, because the students had participated in the norming study, because of technical difficulties, or because more than

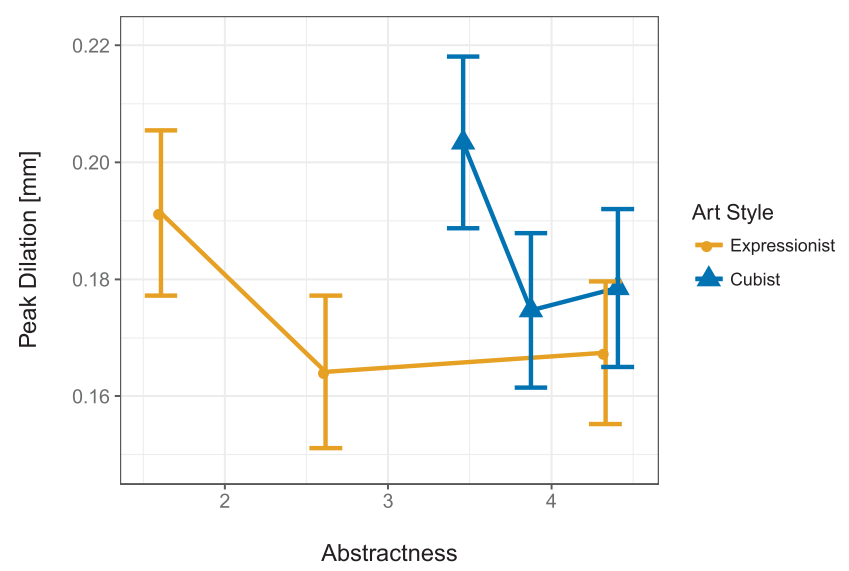

Figure 6. Pupillary peak dilations for the two art styles in Experiment 1. Error bars represent the standard error. See the online article for the color version of this figure.
$50 \%$ of the pupillary data were missing after artifact rejection. The remaining 20 students ( 2 male) were art novices and aged between 18 and 25 years $(M=21.2 ; S D=2.23)$. They had normal or corrected-to-normal vision and received either $5 €$ or course credit as compensation.

Stimuli and procedure. The same stimuli as in the first experiment were used. The procedure was also identical, except that art style was randomly mixed.

The pupillometric data were preprocessed in the same way as in Experiment 1. Altogether, $32 \%$ of the trials (ranging from $12 \%$ to $49 \%$ across participants) were discarded. RT data were handled in the same way as in the previous experiment.

\section{Results and Discussion}

Response times. One-way ANOVAs were computed for each art style with abstractness (low, medium, or high) as withinparticipant factor. A significant effect was found for the expressionist pictures, $F(2,38)=104.4, p<.001 ; \eta^{2}=.606$, as well as for the cubist ones, $F(2,38)=82.2, p<.001 ; \eta^{2}=.411$. As can be seen in Figure 8, RTs again increased with abstractness for both art styles. Subsequent Bonferroni-corrected $t$-tests revealed that RTs differed significantly between all abstractness categories for both art styles, except between expressionist pictures of medium and low abstractness. This result is similar to that observed in Experiment 1.

Correlational analyses. A correlational analysis across the pictures and art styles revealed a significant relation between abstractness and RT $(r=.778 ; p<.001)$. The correlations were also significant for the expressionist pictures alone $(r=.832 ; p<$ $.001)$, as well as for the cubist ones $(r=.794 ; p<.001)$.

Pupillary dilations. Figure 9 shows the response-locked time courses of the pupil size for the different experimental conditions. Different from the previous experiment, pupil size varied monotonically with abstractness for expressionist paintings. The relations were less pronounced for the cubist pictures.

Similar relations were present for the PDs (Figure 10; see also Table 2). One-way ANOVAs revealed, however, that the effect 
Table 2

Mean Response Times (RTs) and Pupillary Peak Dilations (PDs) With Corresponding Standard Deviations in Experiments 1 and 2

\begin{tabular}{|c|c|c|c|c|c|c|c|c|}
\hline \multirow{3}{*}{$\begin{array}{l}\text { Art style and } \\
\text { abstractness }\end{array}$} & \multicolumn{4}{|c|}{ Experiment 1} & \multicolumn{4}{|c|}{ Experiment 2} \\
\hline & \multicolumn{2}{|c|}{$\mathrm{RT}$ (ms) } & \multicolumn{2}{|c|}{$\mathrm{PD}(\mathrm{mm})$} & \multicolumn{2}{|c|}{$\mathrm{RT}(\mathrm{ms})$} & \multicolumn{2}{|c|}{$\mathrm{PD}(\mathrm{mm})$} \\
\hline & $M$ & $S D$ & $M$ & $S D$ & $M$ & $S D$ & $M$ & $S D$ \\
\hline \multicolumn{9}{|l|}{ Expressionist } \\
\hline Low & 1,229 & 950 & .191 & .086 & 1215 & 458 & .279 & .142 \\
\hline Medium & 1,379 & 793 & .164 & .079 & 1551 & 586 & .243 & .083 \\
\hline High & 2,628 & 1436 & .167 & .074 & 3203 & 1000 & .203 & .079 \\
\hline \multicolumn{9}{|l|}{ Cubist } \\
\hline Low & 1,469 & 810 & .203 & .089 & 1722 & 694 & .255 & .128 \\
\hline Medium & 1,992 & 987 & .175 & .080 & 2346 & 776 & .245 & .115 \\
\hline High & 2,703 & 1273 & .179 & .082 & 3277 & 874 & .232 & .087 \\
\hline
\end{tabular}

was significant only for the expressionist pictures, $F(2,38)=5.46$, $p<.01 ; \eta^{2}=.083$, but not for the cubist ones, $F(2,38)=0.745$, $p=.482 ; \eta^{2}=.007$. Subsequent Bonferroni-corrected $t$-tests for the expressionist style showed that the differences in PDs between the low and medium abstractness, and between medium and high abstractness were not significant.

Correlational analyses. The item-based correlational analysis across pictures and art styles revealed that the relation between abstractness and PD was marginally significant $(r=-.215 ; p=$ $.059)$. For the individual art styles, the correlation was significant for the expressionist pictures $(r=-.321 ; p<.05)$, but not for cubist pictures $(r=-.199 ; p=.224)$. Overall, the correlation between RT and PD was also significant $(r=-.266$; $p<.05)$. The faster the responses, the larger the PD. For the individual styles, the correlations were marginally significant for the expressionist pictures (cubist: $r=-.263 ; p=.107$; expressionist: $r=-.308 ; p=.056)$. The overall correlation between preference and PD was not significant $(r=-.077 ; p=.503)$. This also held for the individual styles (cubist: $r=-.040 ; p=.808$; expressionist: $r=-.062 ; p=.706)$. These results show that low abstractness and short RT come along with larger pupil size, as expected. However, there was no indication that PD was related to preference, which is different from the result in Kuchinke et al. (2009).
If we take abstractness into account, then PDs were again larger for cubist pictures than for expressionist ones. To test whether this difference was significant, we applied linear regression to the expressionist data to estimate values for each participant that correspond to the abstractness values of the cubist categories (black squares in Figure 10). The cubist data together with the estimated data for the expressionist pictures were then entered into a one-way ANOVA with art style as within-participant factor. The effect was significant, $F(1$, 19) $=4.51, p<.05 ; \eta^{2}=.027$. Thus, although the effect was small, our data again show a tendency toward greater PDs for cubist pictures than for expressionist stimuli.

Taken together, our results show that, PDs decreased with increasing abstractness of the processed pictures, although the relation was significant only for the expressionist pictures. The item-based overall correlation between PD and RT was significant as well. That is, the faster the responses the larger the measured PDs. These results demonstrate that the mixing of arts styles had a favorable effect on the relation between abstractness and PD. However, there was no itembased correlation between preference and PD, which is different from the results of Kuchinke et al. (2009). Moreover, cubist pictures again produced somewhat larger PDs, given their range of abstractness, which is also contrary to expectation.

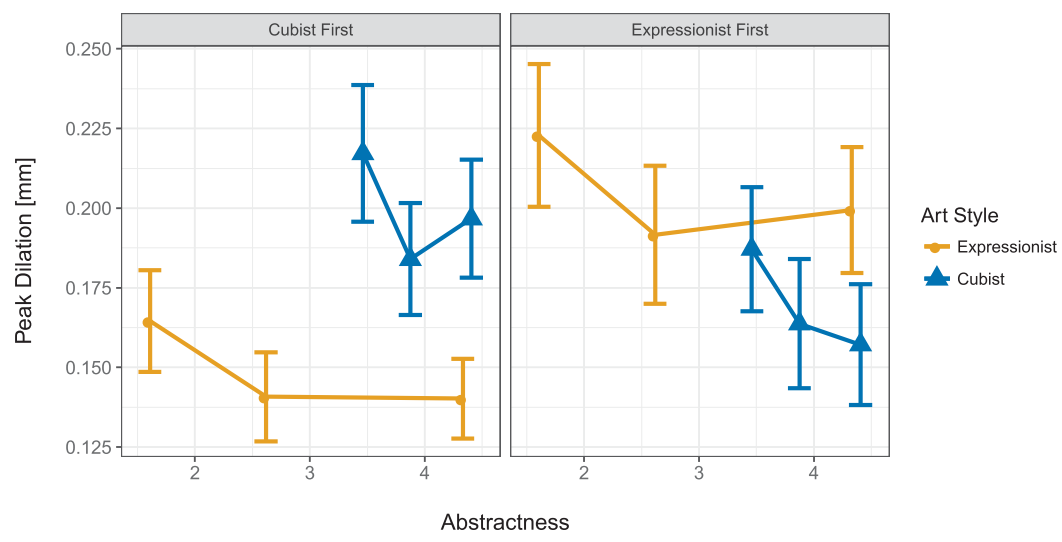

Figure 7. Pupillary peak dilations for the two art styles separated for the two block orders in Experiment 1. Error bars represent the standard error. See the online article for the color version of this figure. 


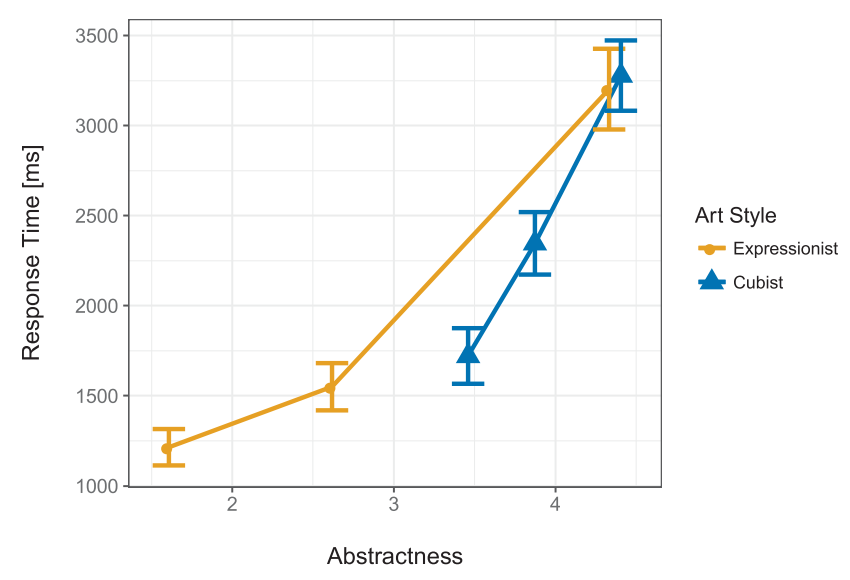

Figure 8. Response times for the cubist and expressionist pictures as function of abstractness in Experiment 2. Error bars represent standard errors. See the online article for the color version of this figure.

\section{General Discussion}

In the present study, we investigated whether the effect of processing fluency on aesthetic preference is also reflected by pupillary responses. Former studies have shown that stimuli that can be processed fluently, are evaluated more positively than stimuli that are difficult to process (e.g., Reber et al., 2004). To account for this result, it has been proposed that fluent processing elicits a positive affect that is interpreted by perceivers as their response to the processed stimulus. To test whether fluency-based affective experience is indeed involved in aesthetic evaluations, Kuchinke et al. (2009) conducted a pupillometric study. In their experiment participants had to recognize an object in cubist paintings as fast as possible. Fluency was assumed to vary with the abstractness of the pictures. As expected, the pupillary PDs increased with decreasing picture abstractness. This result was interpreted in the sense that higher processing fluency increased positive aesthetic affects. Because this is an interesting and im- portant result, the aim of the present study was to replicate the experiment with an improved method and to test whether the observed relations can be generalized to expressionist pictures.

The experimental method was improved in several respects, compared to the study of Kuchinke et al. (2009). For instance, we took measures to avoid confounding the emotional pupil response with the initial light reflex. For generalization, we used expressionist pictures, in addition to the cubist ones in Kuchinke et al. (2009). Pictures of both art styles were rated with respect to preference and abstractness in separate online studies. The results confirmed the relation between fluency (in our case abstractness) and preference, observed in earlier studies (Belke, Leder, Strobach, \& Carbon, 2010; Kuchinke et al., 2009; Reber et al., 1998; Winkielman \& Cacioppo, 2001; Winkielman et al., 2003). In our case, the relation was stronger for the expressionist than for the cubist pictures. A plausible reason for this difference is the fact that expressionist pictures encompassed a larger range of abstractness than the cubist ones. Specifically, the set of expressionist pictures also included stimuli of relatively low abstractness. Their preference ratings were correspondingly higher.

The hypothesis that the relation between preference and abstractness is caused, at least to some extent, by processing fluency, is supported by our result that the object recognition time also increased with abstractness. This was true for both art styles in both experiments, and is in line with previous results (Checkosky \& Whitlock, 1973; Reber et al., 1998; Westerman, Lanska, \& Olds, 2015). Thus, even though RTs might not generally reflect fluency (Oppenheimer, 2008), in our case abstractness clearly determined the speed of processing.

So far, our results suggest that less abstract stimuli are processed more easily, and that fluently processed pictures are associated with higher preference. However, the crucial question is, whether preference is related in any way to fluency-based affective experience, as proposed by Kuchinke et al. (2009). Under the expectation that aesthetic affect is signaled by pupil size, the results of our first experiment are mixed. Although the pupillary PDs were largest for the least abstract pictures, they were numerically larger

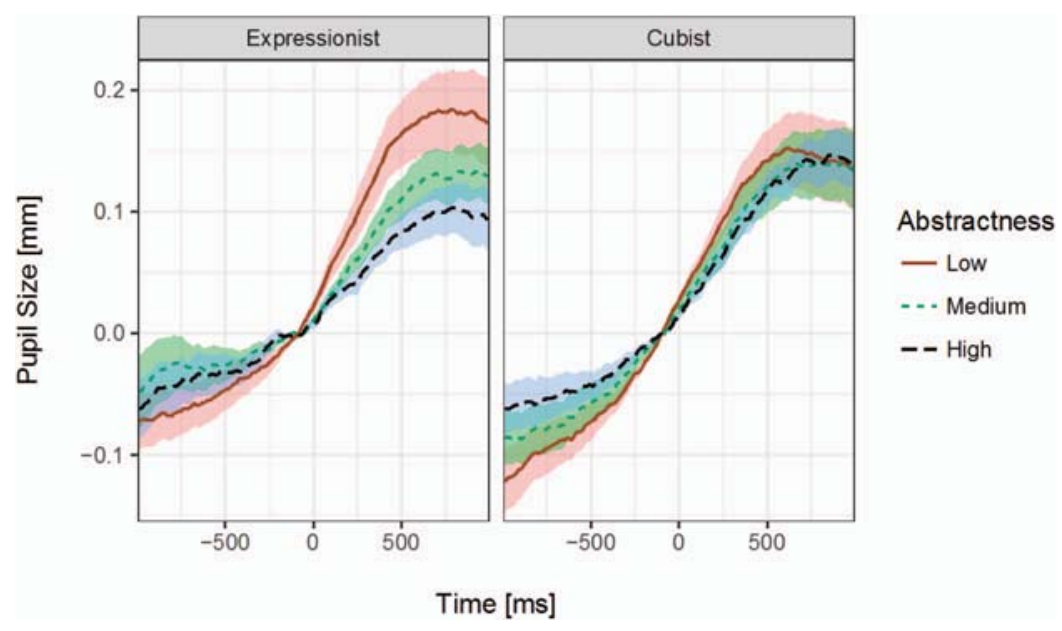

Figure 9. Response-locked time course of the pupil size (in $\mathrm{mm}$ ) for the different art styles and abstractness levels in Experiment 2. Ribbons around the graphs represent standard errors. See the online article for the color version of this figure. 


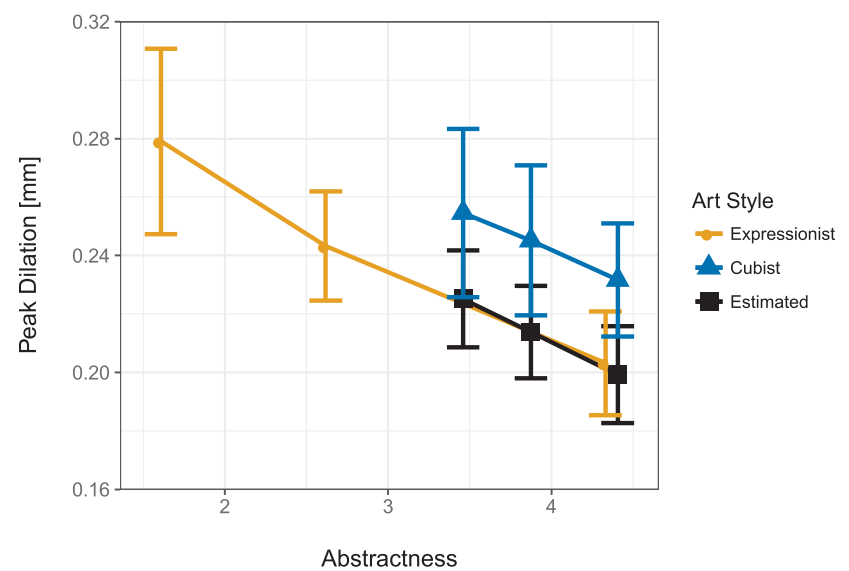

Figure 10. Pupillary peak dilations for the two art styles in Experiment 2. The black squares represent estimated values (by linear regression) of expressionist stimuli that correspond to the range of abstractness values of the cubist stimuli. Error bars represent the standard error. See the online article for the color version of this figure.

for highly than for medium abstract pictures. Moreover, correlational analyses revealed that more preferred pictures produced smaller PDs, which is opposite to the result of Kuchinke et al. (2009). Finally, cubist pictures produced slightly larger PDs than expressionist ones, although the former were more abstract and liked less. Thus, whereas some relations between the variables were in the expected direction, most were opposite.

Especially because art style was blocked, we speculated that this might have contributed to the result that cubist pictures produced larger PDs. Perhaps the participants applied different task sets and response criteria, depending on the art style and their learning history. Therefore, we conducted a second experiment, where the pictures of the two styles were presented randomly across trials.

As a result, randomizing the art style had a positive effect on the relation between abstractness and pupil size. Mean PDs now increased linearly with decreasing abstractness for both art styles (see Figure 10), although the relation was significant only for the expressionist pictures. Thus, strictly speaking, we again failed to replicate the results of Kuchinke et al. (2009), who only applied cubist pictures. One reason for this failure could have been the relatively small range of abstractness for this stimulus set. The small variation of abstractness was probably not sufficient to modulate pupil size substantially. Therefore, it seems reasonable to conclude that easy to process pictures produce larger PDs than difficult to process ones, given a sufficient variation of processing fluency.

However, even if one accepts that processing fluency modulates PDs, does this imply that the PDs also reflect aesthetic affect induced by fluency? Our analyses do not support such a conclusion. Whereas the correlation between preference and PD was negative in Experiment 1, it was absent in our second experiment. Furthermore, despite randomized art style, PDs were again larger for the cubist stimuli than for the expressionist ones, although the former pictures were liked less than the latter ones. One must of course take into account that effect size was rather low. However, the fact that we found this effect in both experiments, shows that it is reliable. Up to now, we have no definitive explanation for the difference in PDs between the art styles. One reviewer suggested that typicality could have played a role. Indeed, if we consider RTs as a valid measure of fluency, then the relation between abstractness and fluency obviously differed between art styles. It could be that cubist pictures were considered as more typical for abstract art than expressionist ones. Consequently, the abstractness of cubist pictures might have been overrated, relative to their actual fluency and compared to expressionist pictures.

Thus, both of our experiments failed to replicate Kuchinke et al.' s (2009) results. For cubist pictures, there were no substantial effects of abstractness on PD. If anything, there were weak tendencies. However, the general idea that abstractness is related to PDs is supported by our results obtained with the expressionist pictures. Thus, it is possible that we did not find an effect for the cubist pictures because of their small range of abstractness.

A more fundamental problem, though, is the reversed (Experiment 1) or absent (Experiment 2) relation between preference and $\mathrm{PD}$, which contradicts the idea that fluency-based aesthetic affect is not only related to preference but also reflected by pupil size. One might argue that we were unable to find a positive relation, because preference ratings and PDs were collected from different samples of participants, respectively, whereas Kuchinke et al. (2009) used a within-participant design. However, this difference should be negligible in our item-based analyses. In fact, we argue that our preference ratings were more representative than Kuchinke et al.'s (2009), because their ratings were obtained after the participants had performed a recognition task. Therefore, it is possible that their participants rated pictures in relation to the ease with which the depicted objects could be recognized rather than regarding its overall aesthetic.

Taken together, our results can hardly be considered as replication of Kuchinke et al.'s (2009) study. Although we observed some relation between the abstractness of art pictures and pupil size during their processing, the relation between pupil size and aesthetic appreciation was absent or reversed. These results do not support the hypothesis that fluency-induced aesthetic affect is reflected by pupil dilation.

\section{References}

Aalto, I. (1917). Autumn landscape with clouds [Painting]. Espoo, Finland: Espoo Museum of Modern Art.

Beatty, J., \& Lucero-Wagoner, B. (2000). The pupillary system. In J. T. Cacioppo, L. G. Tassinary, G. G. Berntson, J. T. Cacioppo, L. G. Tassinary, \& G. G. Berntson (Eds.), Handbook of psychophysiology (2nd ed., pp. 142-162). New York, NY: Cambridge University Press.

Belke, B., Leder, H., Strobach, T., \& Carbon, C. C. (2010). Cognitive fluency: High-level processing dynamics in art appreciation. Psychology of Aesthetics, Creativity, and the Arts, 4, 214-222. http://dx.doi.org/10 $.1037 / \mathrm{a} 0019648$

Blackburn, K., \& Schirillo, J. (2012). Emotive hemispheric differences measured in real-life portraits using pupil diameter and subjective aesthetic preferences. Experimental Brain Research, 219, 447-455. http:// dx.doi.org/10.1007/s00221-012-3091-y

Bornstein, R. F. (1989). Exposure and affect: Overview and meta-analysis of research, 1968-1987. Psychological Bulletin, 106, 265-289. http:// dx.doi.org/10.1037/0033-2909.106.2.265

Bradley, M. M., Miccoli, L., Escrig, M. A., \& Lang, P. J. (2008). The pupil as a measure of emotional arousal and autonomic activation. Psychophysiology, 45, 602-607. http://dx.doi.org/10.1111/j.1469-8986.2008 .00654.x 
Braque, G. (1908). Viaduct at l'Estaque [Painting]. Tel Aviv, Israel: Tel Aviv Museum of Art.

Checkosky, S. F., \& Whitlock, D. (1973). Effects of pattern goodness on recognition time in a memory search task. Journal of Experimental Psychology, 100, 341-348. http://dx.doi.org/10.1037/h0035692

Fechner, G. T. (1876). Vorschule der Aesthetik (Vol. 1). Leipzig, Germany: Breitkopf \& Härtel.

Greiner, B. (2015). Subject pool recruitment procedures: Organizing experiments with ORSEE. Journal of the Economic Science Association, 1, 114-125. http://dx.doi.org/10.1007/s40881-015-0004-4

Gris, J. (1916). Portrait of Josette Gris [Painting]. Madrid, Spain: Museo Reina Sofía.

Hayes, T., Muday, J. A., \& Schirillo, J. A. (2013). Portrait hemispheric laterality measured using pupil diameter and aesthetic judgments. Psychology of Aesthetics, Creativity, and the Arts, 7, 276-284. http://dx.doi .org/10.1037/a0031634

Henderson, R. R., Bradley, M. M., \& Lang, P. J. (2014). Modulation of the initial light reflex during affective picture viewing. Psychophysiology, 51, 815-818. http://dx.doi.org/10.1111/psyp.12236

Jawlensky, A. v. (1912). Self-portrait. Vienna, Austria: Österreichische Galerie Belvedere.

Johnson, M. G., Muday, J. A., \& Schirillo, J. A. (2010). When viewing variations in paintings by Mondrian, aesthetic preferences correlate with pupil size. Psychology of Aesthetics, Creativity, and the Arts, 4, 161167. http://dx.doi.org/10.1037/a0018155

Kiesel, A., Steinhauser, M., Wendt, M., Falkenstein, M., Jost, K., Philipp, A. M., \& Koch, I. (2010). Control and interference in task switching-a review. Psychological Bulletin, 136, 849-874. http://dx.doi.org/10 $.1037 / \mathrm{a} 0019842$

Kuchinke, L., Trapp, S., Jacobs, A. M., \& Leder, H. (2009). Pupillary responses in art appreciation: Effects of aesthetic emotions. Psychology of Aesthetics, Creativity, and the Arts, 3, 156-163. http://dx.doi.org/10 $.1037 / \mathrm{a} 0014464$

Leder, H., Belke, B., Oeberst, A., \& Augustin, D. (2004). A model of aesthetic appreciation and aesthetic judgments. British Journal of Psychology, 95, 489-508. http://dx.doi.org/10.1348/0007126042369811

Loewenfeld, I. E., \& Loewenstein, O. (1993). The pupil: Anatomy, physiology, and clinical applications. Ames, Iowa: Iowa State University Press.

Macke, A. (1913). Girls bathing [Painting]. Private Collection. Retrieved from https://commons.wikimedia.org/wiki/File:August_Macke__Badende_M\%C3\%A4dchen_\%281913\%29.jpg

Malo, J., \& Luque, M. J. (2002). COLORLAB: A color processing toolbox for Matlab. Retrieved from https://www.uv.es/vista/vistavalencia/softw are/colorlab.html

Marc, F. (1914). Landscape with house, dog and cattle [Painting]. Küsnacht, Switzerland. Private Collection.

Oppenheimer, D. M. (2008). The secret life of fluency. Trends in Cognitive Sciences, 12, 237-241. http://dx.doi.org/10.1016/j.tics.2008.02.014

Palmer, S. E., Schloss, K. B., \& Sammartino, J. (2013). Visual aesthetics and human preference. Annual Review of Psychology, 64, 77-107. http://dx.doi.org/10.1146/annurev-psych-120710-100504

Partala, T., \& Surakka, V. (2003). Pupil size variation as an indication of affective processing. International Journal of Human-Computer Studies, 59, 185-198. http://dx.doi.org/10.1016/S1071-5819(03)00017-X
Popova, L. (1913). Composition with figures [Painting]. Moscow, Russia: State Tretyakow Gallery.

Powell, W. R., \& Schirillo, J. A. (2011). Hemispheric laterality measured in Rembrandt's portraits using pupil diameter and aesthetic verbal judgements. Cognition and Emotion, 25, 868-885. http://dx.doi.org/10.1080/ 02699931.2010 .515709

R Core Team. (2015). R: A language and environment for statistical computing. Vienna, Austria: R Foundation for Statistical Computing. Retrieved from http://www.R-project.org

Reber, R., Schwarz, N., \& Winkielman, P. (2004). Processing fluency and aesthetic pleasure: Is beauty in the perceiver's processing experience? Personality and Social Psychology Review, 8, 364-382. http://dx.doi .org/10.1207/s15327957pspr0804_3

Reber, R., Winkielman, P., \& Schwarz, N. (1998). Effects of perceptual fluency on affective judgments. Psychological Science, 9, 45-48. http:// dx.doi.org/10.1111/1467-9280.00008

Siegle, G. J., Ichikawa, N., \& Steinhauer, S. (2008). Blink before and after you think: Blinks occur prior to and following cognitive load indexed by pupillary responses. Psychophysiology, 45, 679-687. http://dx.doi.org/ 10.1111/j.1469-8986.2008.00681.x

Thomas, K. (2000). DuMont's Kunstlexikon des 20. Jahrhunderts: Künstler, Stile [DuMont's art dictionary of the 20th century: artists, styles]. DuMont: Begriffe.

Võ, M. L. H., Jacobs, A. M., Kuchinke, L., Hofmann, M., Conrad, M., Schacht, A., \& Hutzler, F. (2008). The coupling of emotion and cognition in the eye: Introducing the pupil old/new effect. Psychophysiology, $45,130-140$.

Westerman, D. L., Lanska, M., \& Olds, J. M. (2015). The effect of processing fluency on impressions of familiarity and liking. Journal of Experimental Psychology: Learning, Memory, and Cognition, 41, 426438. http://dx.doi.org/10.1037/a0038356

Wickham, H. (2009). ggplot2: Elegant graphics for data analysis: New York, NY: Springer.

Winkielman, P., \& Cacioppo, J. T. (2001). Mind at ease puts a smile on the face: Psychophysiological evidence that processing facilitation elicits positive affect. Journal of Personality and Social Psychology, 81, 9891000. http://dx.doi.org/10.1037/0022-3514.81.6.989

Winkielman, P., Schwarz, N., Fazendeiro, T., \& Reber, R. (2003). The hedonic marking of processing fluency: Implications for evaluative judgment. In J. Musch, K. C. Klauer (Eds.), The psychology of evaluation: Affective processes in cognition and emotion (pp. 189-217). Mahwah, NJ: Lawrence Erlbaum Associates Publishers.

Woodmansee, J. J. (1966). Methodological problems in pupillographic experiments. In Proceedings of the Annual Convention of the American Psychological Association (pp. 133-134). Washington, DC: American Psychological Association.

Zajonc, R. B., Markus, H., \& Wilson, W. R. (1974). Exposure effects and associative learning. Journal of Experimental Social Psychology, 10, 248-263. http://dx.doi.org/10.1016/0022-1031(74)90071-7 